\title{
AXISYMMETRIC BIFURCATION IN AN ELASTIC-PLASTIC CYLINDER UNDER AXIAL LOAD AND LATERAL HYDROSTATIC PRESSURE*
}

BY

\author{
S. Y. CHENG, S. T. ARIARATNAM AND R. N. DUBEY
}

University of Waterloo

Summary. Conditions for initiation of necking and bulging of elastic and elasticplastic cylindrical solids are derived. The possibility of bifurcation of rigid-plastic solids and the conditions for homogeneous deformation with homogeneous stress are also investigated.

1. Introduction. This paper deals with some problems of bifurcation in cylindrical solids of elastic, rigid-plastic and elastic-plastic material. One particular objective of this study is to investigate the effect of hydrostatic pressure on the necking and bulging loads of a specimen in tension and compression tests. The constitutive laws assumed for the different materials are those due to Hill [3].

Previous works related to the problem concerned have been confined either to the necking of an elastic cylinder under uniaxial stress (Wesolowski [10]) or to the stability of elastic rectangular solids subjected to pressure loads (Wu and Widera [11] and Kerr and Tang [5], [6]). The effect of hydrostatic pressure on the necking load of plastic solids has long been a point of controversy. However, for rigid-plastic material, Hill [2] first proved that lateral pressure does not affect the tensile necking load. A similar conclusion was reached by Pugh [8] and Alexander [1], but their arguments are unsatisfactory. The results of the present investigation provide a rigorous confirmation of this result for an incompressible elastic-plastic cylinder.

2. Formulation of the problem. Consider an incompressible cylindrical solid of elastic-plastic material subjected to an axial load $P$ and lateral hydrostatic pressure of intensity $q$. Suppose that the body undergoes finite homogeneous deformation due to $P$ and $q$ from some initial state $B^{0}$ to the current state $B$. The deformed body in the state $B$ is supposed to preserve a cylindrical shape with radius $a$ and length $2 l$; the necessary information for complete description of $B$ is assumed given. To investigate the bifurcation of equilibrium for continuing deformation, the behaviour of the specimen in transition from $B$ to a neighbouring state $B^{\prime}$, under the infinitesimal increments of the boundary values, is isolated for study. Only axially symmetric deformation will be considered in this paper.

Referred to state $B$, a fixed cylindrical coordinate system $x_{i}$, with axes coinciding with axes of symmetry of the specimen, is taken as the reference frame. Whenever convenient the coordinates $x_{1}, x_{2}, x_{3}$ will be replaced by $r, \theta, z$ respectively and the velocity components $v_{1}, v_{2}, v_{3}$ by $u, v, w$ respectively. In the current state, we have

* Received January 2, 1970; revised version received July 29, 1970. 


$$
g_{i j}=\left(\begin{array}{ccc}
1 & 0 & 0 \\
0 & r^{2} & 0 \\
0 & 0 & 1
\end{array}\right), \quad g^{i j}=\left(\begin{array}{ccc}
1 & 0 & 0 \\
0 & r^{-2} & 0 \\
0 & 0 & 1
\end{array}\right)
$$

where $g_{i j}$ and $g^{i i}$ are the covariant and the contravariant components of the metric tensor. The internal distribution of stress, assumed homogeneous, may then be supposed given by

$$
\sigma^{i j}=s^{i i}=\left(\begin{array}{ccc}
-q & 0 & 0 \\
0 & -q r^{-2} & 0 \\
0 & 0 & -p
\end{array}\right)
$$

where $\sigma^{i s}, s^{i i}$ are respectively, the contravariant components of the true stress and the nominal stress tensor; $p=P / \pi a^{2}$.

The stress distribution (2) satisfies the equations of equilibrium:

$$
\sigma_{, i}^{i i}=0
$$

where the comma denotes the covariant derivative, and the boundary conditions:

$$
\begin{aligned}
& T^{i}=(-q, 0,0) \text { for } r=a \\
& =(0,0, \mp p) \text { for } z= \pm l
\end{aligned}
$$

where $T^{\prime}$ is the surface traction.

For the continuing deformation from $B$ to $B^{\prime}$, the longitudinal ends $z= \pm l$, moving with constant velocity $\mp w_{0}$, are assumed to be frictionless (hence the shear traction rates on these ends are zero) and the rate of hydrostatic pressure is specified as $-\dot{q}$. Using the nominal traction rate $\dot{T}^{i}$ (see, e.g. Hill [3], [4]) and remembering that the velocity field is axially symmetric, we have

$$
\begin{gathered}
w=\mp w_{0}, \quad \dot{T}^{1}=n_{i} \dot{S}^{i 1}=0 \text { at } z= \pm l, \\
\dot{T}^{i}=\dot{s}^{i j} n_{i}=-\dot{q} n^{i}-q\left(v^{k}{ }_{, k} n^{i}-g^{i k} n^{i} v_{i, k}\right) \quad \text { for } \quad r=a
\end{gathered}
$$

where $n_{i}$ and $n^{i}$ are respectively the covariant and the contravariant components of the unit outward normal to the boundary surface; $\dot{s}^{i i}$ is the material derivative of the nominal stress tensor which is related to the material derivative of the true stress tensor $\dot{\sigma}^{i i}$ by (see, e.g., Hill [3])

$$
\dot{s}^{i j}=\dot{\sigma}^{i j}+\sigma^{i j} v_{, k}^{k}-\sigma^{i k} v_{, k}^{i},
$$

where the operator of material derivative is defined as

$$
\text { ( ) }=\frac{\partial()}{\partial t}+v^{k}()_{. k} \text {. }
$$

Using (5) and the incompressibility condition $v_{, k}^{k}=0$, the boundary conditions (4) reduce to

$$
\begin{array}{ccc}
w=\mp w_{0}, \quad \dot{\sigma}^{2 r}=-q_{w_{r}}=0 \quad \text { at } & z= \pm l, \\
\dot{\sigma}^{r r}=-\dot{q}, \quad \dot{\sigma}^{r z}=-(p-q) u_{z} & \text { at } \quad r=a,
\end{array}
$$

where $u_{z}$ denotes partial differentiation with respect to $z$. 
In view of (2), (3) and (5), the equations of continuing equilibrinm $\dot{s}_{. i}^{i j}=0$ become

$$
\dot{\sigma}_{. i}^{i j}=0
$$

or equivalently, in terms of partial derivatives,

$$
\begin{gathered}
\partial \dot{\sigma}^{r r} / \partial r+\partial \dot{\sigma}^{r z} / \partial z+\dot{\sigma}^{r r} / r-r \dot{\sigma}^{\theta \theta}=0, \\
\partial \dot{\sigma}^{r z} / \partial r+\partial \dot{\sigma}^{z z} / \partial z+\dot{\sigma}^{r z} / r=0 .
\end{gathered}
$$

The equation for $j=2$ is identically satisfied for the case of axially symmetric deformation considered here.

Material properties. For axially symmetric deformation, $u=u(r, z), v=0$ and $w=w(r, z)$. Hence the rate of deformation tensor $\epsilon_{i j}=\frac{1}{2}\left(v_{i, j}+v_{i, i}\right)$ and the spin tensor $\omega_{i j}=\frac{1}{2}\left(v_{i, i}-v_{i, i}\right)$ can be easily obtained and are as follows:

$$
\begin{aligned}
\epsilon_{i j} & =\left[\begin{array}{ccc}
u_{r} & 0 & \frac{1}{2}\left(u_{z}+w_{r}\right) \\
0 & r u & 0 \\
\frac{1}{2}\left(u_{z}+w_{r}\right) & 0 & w_{z}
\end{array}\right], \\
\omega_{i j} & =\left[\begin{array}{ccc}
0 & 0 & \frac{1}{2}\left(u_{z}-w_{r}\right) \\
0 & 0 & 0 \\
-\frac{1}{2}\left(u_{z}-w_{r}\right) & 0 & 0
\end{array}\right] .
\end{aligned}
$$

Furthermore, the condition of incompressibility reduces to

$$
u_{r}+u / r+w_{z}=0 .
$$

The rate constitutive equation for the material is taken in the form proposed by Hill [3], the objective stress rate used being the Jaumann derivative of the true stress tensor, which vanishes under rigid body rotation. This stress rate, denoted by $D \sigma^{i j} / D t$, is related to the material derivative $\dot{\sigma}^{i i}$ by

$$
D \sigma^{i j} / D t=\dot{\sigma}^{i i}-\sigma^{i k} \omega^{i}{ }_{k}-\sigma^{k i} \omega^{i}{ }_{k} .
$$

For linear, isotropic and incompressible material, the constitutive equations become

Elastic material:

$$
D \sigma^{i i} / D t=2 \mu \epsilon^{i j}-g^{i j} \dot{q}^{\prime}
$$

Rigid-plastic material:

$$
\begin{aligned}
\epsilon_{i j} & =h^{-1} m_{i j}\left(m_{k l}\left(D \sigma^{k l} / D t\right)\right) & \text { when } & m_{k l}\left(D \sigma^{k l} / D t\right)>0, \\
& =0 & \text { when } & m_{k l}\left(D \sigma^{k l} / D t\right) \leq 0 .
\end{aligned}
$$

As will be shown in the appendix, it is immaterial whether the Jaumann derivative or the material derivative is used in this relation.

Elastic-plastic material:

$$
\begin{aligned}
& D \sigma^{i i} / D t=2 \mu \epsilon^{i j}-\left(4 \mu^{2} /(2 \mu+h)\right)\left(m_{k l} \epsilon^{k l}\right) m^{i j}-g^{i j} \dot{q}^{\prime} \text { when } m_{k l}\left(D \sigma^{k l} / D t\right)>0 \text {, } \\
& =2 \mu \epsilon^{i j}-g^{i j} \dot{q}^{\prime} \quad \text { when } m_{k l}\left(D \sigma^{k l} / D t\right) \leq \mathbf{0} .
\end{aligned}
$$

where $\mu$ is Lamé's constant, $\dot{q}^{\prime}$ is an unknown scalar function, $h$ is a positive scalar measure 
of the current rate of work-hardening, and $m_{i i}, m^{i i}$ are covariant and contravariant components of the unit outward normal to the local yield surface in six-dimensional stress space.

In view of the form of the current stress distribution (2), the components of $m_{i j}$ and $m^{i j}$ may be taken in the form

$$
\begin{aligned}
m_{i i} & =\left[\begin{array}{ccc}
m_{1} & 0 & 0 \\
0 & m_{2} r^{2} & 0 \\
0 & 0 & m_{3}
\end{array}\right], \\
m^{i j} & =\left[\begin{array}{ccc}
m_{1} & 0 & 0 \\
0 & m_{2} r^{-2} & 0 \\
0 & 0 & m_{3}
\end{array}\right],
\end{aligned}
$$

where

$$
m_{1}+m_{2}+m_{3}=0 \quad \text { (incompressibility) and } m_{1}^{2}+m_{2}^{2}+m_{3}^{2}=1 .
$$

Furthermore, since $\sigma^{\theta \theta}=\sigma^{r r}=-q$, we must have $m_{1}=m_{2}$. This together with (16) completely determines the components of $m_{i i}$, which is found to be that corresponding to the von Mises criterion.

3. Bifurcations in elastic solids. The solution of the linear system represented by (6), (7), (10), and (11) can be considered as a result of superposition of the following deformations:

(i) Homogeneous deformation with boundary conditions given by

$$
\begin{gathered}
w=\mp w_{0}, \quad \dot{\sigma}^{r s}=0 \quad \text { at } \quad z= \pm l \\
\dot{\sigma}^{r r}=-\dot{q}, \quad \dot{\sigma}^{r z}=0 \quad \text { at } \quad r=a .
\end{gathered}
$$

(ii) Nonhomogeneous deformation with boundary conditions given by

$$
\begin{aligned}
& w=0, \quad \dot{\sigma}^{r z}=0 \quad \text { at } \quad z= \pm l, \\
& \dot{\sigma}^{r r}=0, \quad \dot{\sigma}^{r z}=-(p-q) u_{z} \quad \text { at } \quad r=a .
\end{aligned}
$$

Evidently the solution associated with (i) is also a complete solution which satisfies the boundary conditions (6), since $u_{z}$ vanishes identically for homogeneous deformation. A nontrivial solution associated with (ii), which exists only for some particular values of $\sigma^{i s}$, corresponds to bifurcation of equilibrium. The superposition of the two solutions is another complete solution. The bifurcation will correspond to either necking or bulging, depending on whether the current axial stress is tensile or compressive.

Homogeneous deformation. A solution of (7) which satisfies the boundary conditions (6a) and the incompressibility condition (10) may be found to be

$$
\begin{aligned}
u & =\left(w_{0} / 2\right)(r / l), & w & =-\left(w_{0} / l\right) z, \\
\dot{\sigma}^{r r} & =-\dot{q}, & \dot{\sigma}^{\theta \theta} & =-r^{-2} \dot{q}, \\
\dot{\sigma}^{2 z} & =-3 \mu\left(w_{0} / l\right)-\dot{q}, & \dot{\sigma}^{r z} & =0,
\end{aligned}
$$

and the corresponding rate of loading on the faces $z= \pm l$ is

$$
\dot{T}^{3}=-(3 \mu+p)\left(w_{0} / l\right)-\dot{q}
$$


This solution preserves the cylindrical shape of specimen with uniform distribution of $\dot{\sigma}^{i j}$. Hence the previous assumption of a homogeneous current stress field is justified, since it is attainable by a succession of incremental deformations from the state $B^{0}$.

Nonhomogeneous deformation. In view of (10) and the boundary conditions (6b), we seek a solution of the form

$$
u=f_{1}(r) \cos \nu z, \quad w=f_{2}(r) \sin \nu z, \quad \dot{q}^{\prime}=f_{3}(r) \cos \nu z,
$$

where $\nu=n \pi / l, n$ being an integer, and $f_{i}(r)$ are unknown functions to be determined. Substituting (19) in (12) and then eliminating $f_{2}$ and $f_{3}$, using (1), (2), (7), (10), and (11), we finally arrive at

$$
\left(L^{4}+2 \nu^{2} b L^{2}+\nu^{4} C\right) f_{1}=0,
$$

where

$$
L^{2}=\frac{d}{d r}\left[\frac{1}{r} \frac{d}{d r}\left(r_{.}\right)\right]
$$

and

$$
b=-\frac{1}{1+\phi}, \quad C=\frac{1-\phi}{1+\phi}
$$

with

$$
\phi=(p-q) / 2 \mu .
$$

Similarly, using (1), (2), (11), (12) and (19), the boundary conditions (6b) reduce to

$$
\begin{aligned}
w=0, \quad u_{s}=0 \quad \text { at } z= \pm l & \\
\frac{d}{d r}\left(r L^{2} f_{1}\right)+2 \nu^{2}\left(b-\frac{1}{2}\right) r \frac{d}{d r} f_{1}-\nu^{2} f_{1}=0, & \left(L^{2}+\nu^{2}\right) f_{1}=0 \quad \text { at } \quad r=a .
\end{aligned}
$$

It can be seen that (6c) is identically satisfied by (19).

Eq. (20) can be written in the form

$$
\left(L^{2}+\nu^{2} \kappa_{1}^{2}\right)\left(L^{2}+\nu^{2} \kappa_{2}^{2}\right) f_{1}=0
$$

where $\kappa_{1}$ and $\kappa_{2}$ are functions of $\phi$ (complex in general) and can be represented as

$$
\kappa_{1}, \kappa_{2}=\left[b \pm \sqrt{ }\left(b^{2}-c\right)\right]^{1 / 2}
$$

Two distinct cases may arise: (i) $\kappa_{1} \neq \kappa_{2}$ corresponding to $b^{2}-c \neq 0$, (ii) $\kappa_{1}=\kappa_{2}$ corresponding to $b^{2}-c=0$.

(i) $\kappa_{1} \neq \kappa_{2}$ : The general solution of (21) may be written as

$$
f_{1}=A_{1} J_{1}\left(\nu \kappa_{1} r\right)+A_{2} J_{1}\left(\nu \kappa_{2} r\right)+A_{3} Y_{1}\left(\nu \kappa_{1} r\right)+A_{4} Y_{1}\left(\nu \kappa_{2} r\right)
$$

where $A_{i}$ are integration constants and $J_{1}$ and $Y_{1}$ are the Bessel functions of the first and second kind, respectively. Since $f_{1}(r)$ should be finite at $r=0, A_{3}$ and $A_{1}$ must both vanish, and (22) reduces to

$$
f_{1}=A_{1} J_{1}\left(\nu \kappa_{1} r\right)+A_{2} J_{1}\left(\nu \kappa_{2} r\right)
$$

The constants $A_{1}$ and $A_{2}$ must be determined from the boundary conditions (6d); 
thus, by substitution, we obtain

$$
\begin{aligned}
{\left[\frac{2}{1+\phi} J_{1}\left(\nu \kappa_{1} a\right)-\right.} & \left.\nu \kappa_{1} a\left(1+\kappa_{1}^{2}+\frac{2}{1+\phi}\right) J_{0}\left(\nu \kappa_{1} a\right)\right] A_{1} \\
+ & {\left[\frac{2}{1+\phi} J_{1}\left(\nu \kappa_{2} a\right)-\nu \kappa_{2} a\left(1+\kappa_{2}^{2}+\frac{2}{1+\phi}\right) J_{0}\left(\nu \kappa_{2} a\right)\right] A_{2}=0 } \\
& {\left[\left(1-\kappa_{1}^{2}\right) J_{1}\left(\nu \kappa_{1} a\right)\right] A_{1}+\left[\left(1-\kappa_{2}^{2}\right) J_{1}\left(\nu \kappa_{2} a\right)\right] A_{2}=0 . }
\end{aligned}
$$

The condition for bifurcation is that there exist a nontrivial solution of (23); i.e., the characteristic determinant of the coefficients is equal to zero. Since $\kappa_{1}$ and $\kappa_{2}$ may be either real or imaginary, it is convenient to deal with the problem in the following separate cases:

(a) $\kappa_{1}, \kappa_{2}$ real, $b^{2}-c>0, b>0$. The condition for bifurcation can be immediately obtained from (23) as:

$$
\frac{\left(1-\kappa_{1}^{2}\right) J_{1}\left(\nu \kappa_{1} a\right)}{\left(1-\kappa_{2}^{2}\right) J_{1}\left(\nu \kappa_{2} a\right)}=\frac{2 J_{1}\left(\nu \kappa_{1} a\right)-\nu \kappa_{1} a\left[\left(1+\kappa_{1}^{2}\right)(1+\phi)+2\right]}{2 J_{1}\left(\nu \kappa_{2} a\right)-\nu \kappa_{2} a\left[\left(1+\kappa_{2}^{2}\right)(1+\phi)+2\right]} \frac{J_{0}\left(\nu \kappa_{1} a\right)}{J_{0}\left(\nu \kappa_{2} a\right)}
$$

(b) $\kappa_{1}, \kappa_{2}$ imaginary, $b^{2}-c>0, b<0$. Denoting

$$
\chi_{1}=i_{\kappa_{1}}, \chi_{2}=i_{\kappa_{2}}
$$

we have, instead of (21), (22a) and (24)

$$
\begin{gathered}
\left(L^{2}-\nu^{2} \chi_{1}^{2}\right)\left(L^{2}-\nu^{2} \chi_{2}^{2}\right) f_{1}=0 \\
f_{1}=A_{1} I_{1}\left(\nu \chi_{1} r\right)+A_{2} I_{1}\left(\nu \chi_{2} r\right) \\
\frac{\left(1+\chi_{1}^{2}\right) I_{1}\left(\nu \chi_{1} a\right)}{\left(1+\chi_{2}^{2}\right) I_{1}\left(\nu \chi_{2} a\right)}=\frac{2 I_{1}\left(\nu \chi_{1} a\right)-\nu \chi_{1} a\left[\left(1-\chi_{1}^{2}\right)(1+\phi)+2\right] I_{0}\left(\nu \chi_{1} a\right)}{2 I_{1}\left(\nu \chi_{2} a\right)-\nu \chi_{2} a\left[\left(1-\chi_{2}^{2}\right)(1+\phi)+2 j I_{0}\left(\nu \chi_{2} a\right)\right.}
\end{gathered}
$$

where $I_{1}$ and $I_{0}$ are modified Bessel functions of the 1st kind.

(c) $\kappa_{1}$ real, $\kappa_{2}$ imaginary, $b^{2}-c>0, c<0$. In this case, the condition for bifurcation becomes

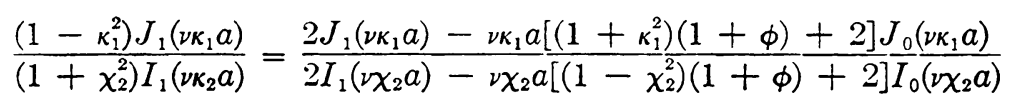

(ii) $\kappa_{1}=\kappa_{2}=i$, corresponding to $p=q$. The general solution of (21), nonsingular at $r=0$, now becomes

$$
f_{1}=A_{1} I_{1}(\nu r)+A_{2} r I_{0}(\nu r)
$$

A similar calculation leads to the following condition for bifurcation:

$$
\nu^{2} a^{2} I_{1}^{2}(\nu a)+\left[\nu a I_{0}(\nu a)-I_{1}(\nu a)\right]^{2}=0 .
$$

This implies that the characteristic determinant of the coefficients in (23) does not vanish unless $\nu a$ vanishes. In other words, no bifurcation can occur for $p=q$.

For any material for which $\mu$ is given, the critical stress for bifurcation can be determined from (24)-(26).

For a material for which $|(p-q) / 2 \mu|<1$ (for instance, most of metals and brittle materials), we have $b\left\langle 0, c>0\right.$ and $b^{2}-c=(\phi /(1+\phi))^{2}>0$. The condition for 
bifurcation (25) leads to

$$
S(\nu a)-(1+\phi)^{2} S\left[\nu\left(\frac{1-\phi}{1+\phi}\right)^{1 / 2} a\right]+\phi=0
$$

where

$$
S(x)=x I_{0}(x) / I_{1}(x) \text {. }
$$

For constant $\mu$ and a finite value of $a / l,(29)$ is satisfied only when $\phi>0$. In other words, the specimen can bulge if $p>q$. However, no necking is possible for specimen under tension, with or without lateral hydrostatic pressure.

For $a / l \ll 1,(24)$ reduces to

$$
(p-q)_{c} \doteq-3 \mu\left(1+\frac{1}{8} \frac{n^{2} \pi^{2} a^{2}}{l^{2}}\right) .
$$

Since the effective tangent modulus is $3 \mu$, it can be seen that the first term gives the usual engineering solution while the second term represents the correction due to shear stiffening.

It should be pointed out that for elastic materials with constant $\mu$, bifurcation occurs when the difference between $p$ and $q, p-q$, reaches some critical value. Hence in tension and compression tests with the specimen subjected to an all-round hydrostatic pressure, the critical load (the load applied by the machine head) is independent of the hydrostatic pressure. If $\mu$ varies with stress, it would be expected to be a function of the current stress deviator. For the case considered here, all the nonvanishing components of current stress deviator are in terms of $(p-q)$; hence the aforementioned conclusion would still hold.

4. Bifurcations in rigid-plastic solids. Assuming that the material loads everywhere, we rewrite (13), in view of (8) and (15), in the form

$$
u_{r}=\lambda m_{1}, \quad u / r=\lambda m_{2}, \quad w_{z}=\lambda m_{3}, \quad u_{z}+w_{r}=0
$$

where $\lambda=h^{-1}\left(m_{i j} \dot{\sigma}^{i j}\right)$ is a scalar function depending on the position. For von Mises material $m_{1}=m_{2}=1 / \sqrt{ } 6, m_{3}=-2 / \sqrt{ } 6$.

By a procedure similar to that of Sec. 3, we seek solutions of (30) and (7) which separately satisfy the boundary conditions (6a) and (6b).

Homogeneous deformation. A solution corresponding to homogeneous deformation is found to be

$$
\begin{aligned}
u & =\frac{1}{2} \frac{w_{0}}{l} r, & w & =-\frac{w_{0}}{l} z, \\
\dot{\sigma}^{r r} & =-\dot{q}, & \dot{\sigma}^{\theta \theta} & =-r^{-2} \dot{q}, \\
\dot{\sigma}^{z s} & =-\frac{3}{2} h \frac{w_{0}}{l}-\dot{q}, & \dot{\sigma}^{r z} & =0,
\end{aligned}
$$

and the corresponding rate of loading on the faces $z= \pm l$ is

$$
\dot{T}^{3}=-((3 / 2) h+p)-\dot{q}
$$

Hence the assumption of homogeneous current stress field is again justified.

Nonhomogeneous deformation. In view of the components of $m_{i j}$ for von Mises 
material, (30) can be written as

$$
u_{r}=u / r=-w_{z} / 2=\lambda / \sqrt{ } 6, \quad u_{z}+w_{r}=0 .
$$

Similar to the result obtained by Prager [7], the general solution of $\left(30^{\prime}\right)$ is found to be

$$
\begin{aligned}
& u=c_{1} r z+c_{2} r, \\
& w=-\frac{c_{1}}{2} r^{2}-c_{1} z^{2}-2 c_{2} z+c_{3}, \\
& \lambda=\sqrt{ } 6\left(c_{1} z+c_{2}\right)
\end{aligned}
$$

where $c_{i}$ are arbitrary constants. It is worth noting that this solution involves no arbitrary function. By symmetry and from the boundary conditions, $w=0$, for $z= \pm l$, we have $c_{1}=c_{2}=c_{3}=0$; hence $u=w \equiv 0$.

This implies that in the case of rigid-plastic materials the possibility of necking or bulging deformations is ruled out by the equations of compatibility alone.

5. Bifurcations in elastic-plastic solids. As in the case of elastic solids, a similar calculation for elastic-plastic material, again assumed to load everywhere, leads to the following results:

Homogeneous deformation. A solution corresponding to homogeneous deformation is found to be

$$
\begin{aligned}
u & =\frac{1}{2} \frac{w_{0}}{l} r, & w & =-\frac{w_{0}}{l} z, \\
\dot{\sigma}^{r r} & =-\dot{q}, & \dot{\sigma}^{\theta \theta} & =-r^{-2} \dot{q}, \\
\dot{\sigma}^{z z} & =-\frac{3 \mu}{1+\delta} \frac{w_{0}}{l}-\dot{q}, & \dot{\sigma}^{r z} & =0
\end{aligned}
$$

where $\delta=2 \mu / h$, and the corresponding rate of loading on the faces $z= \pm l$ is

$$
\dot{T}^{3}=-\left(\frac{3 \mu}{1+\delta}+p\right) \frac{w_{0}}{l}-\dot{q}
$$

This solution also preserves the cylindrical shape of the specimen, and the assumption of homogeneous current stress field is again justified.

Eqs. (33a) and (33b) reduce to (17) and (18) respectively when $h$ approaches infinity, i.e. when the material is elastic.

Nonhomogeneous deformation. The quantities $\kappa_{1}, \kappa_{2}$ in (21a) now become

$$
\kappa_{1}, \kappa_{2}=\left[b \pm \sqrt{ }\left(b^{2}-c\right)\right]^{1 / 2}
$$

where

$$
b=-\frac{2-\delta}{2(1+\phi)(1+\delta)}, \quad c=\frac{1-\phi}{1+\phi},
$$

and, the conditions for bifurcation for the case of elastic-plastic material obeying von Mises criterion are obtained as

(i) $\kappa_{1} \neq \kappa_{2}$ :

$$
\begin{aligned}
& \text { (a) } \kappa_{1}, \kappa_{2} \text { real, } \\
& \frac{\left(1-\kappa_{1}^{2}\right) J_{1}\left(\nu \kappa_{1} a\right)}{\left(1-\kappa_{2}^{2}\right) J_{1}\left(\nu \kappa_{2} a\right)}=\frac{2 J_{1}\left(\nu \kappa_{1} a\right)-\nu \kappa_{1} a\left[(1+\phi)\left(1+\kappa_{1}^{2}\right)+(2-\delta) /(1+\delta)\right] J_{0}\left(\nu \kappa_{1} a\right)}{2 J_{1}\left(\nu \kappa_{2} a\right)-\nu \kappa_{2} a\left[(1+\phi)\left(1+\kappa_{2}^{2}\right)+(2-\delta) /(1+\delta)\right] J_{0}\left(\nu \kappa_{2} a\right)} .
\end{aligned}
$$


(b) $\kappa_{1}, \kappa_{2}$ imaginary, $\chi_{i}=i_{\kappa_{i}}$,

$$
\frac{\left(1+\chi_{1}^{2}\right) I_{1}\left(\nu \chi_{1} a\right)}{\left(1+\chi_{2}^{2}\right) I_{1}\left(\nu \chi_{2} a\right)}=\frac{2 I_{1}\left(\nu \chi_{1} a\right)-\nu \chi_{1} a\left[(1+\phi)\left(1-\chi_{1}^{2}\right)+(2-\delta) /(1+\delta)\right] I_{0}\left(\nu \chi_{1} a\right)}{2 I_{1}\left(\nu \chi_{2} a\right)-\nu \chi_{2} a\left[(1+\phi)\left(1-\chi_{2}^{2}\right)+(2-\delta) /(1+\delta)\right] I_{0}\left(\nu \chi_{2} a\right)} .
$$

(c) $\kappa_{1}$ real, $\kappa_{2}$ imaginary,

$$
\frac{\left(1-\kappa_{1}^{2}\right) J_{1}\left(\nu \kappa_{1} a\right)}{\left(1+\chi_{2}^{2}\right) I_{1}\left(\nu \chi_{2} a\right)}=\frac{2 J_{1}\left(\nu \kappa_{1} a\right)-\nu \kappa_{1} a\left[(1+\phi)\left(1+\kappa_{1}^{2}\right)+(2-\delta) /(1+\delta)\right] J_{0}\left(\nu \kappa_{1} a\right)}{2 I_{1}\left(\nu \chi_{2} a\right)-\nu \chi_{2} a\left[(1+\phi)\left(1-\chi_{2}^{2}\right)+(2-\delta) /(1+\delta)\right] I_{0}\left(\nu \chi_{2} a\right)} \text {. }
$$

(d) $\kappa_{1}, \kappa_{2}$ conjugate complex, $b^{2}-c<0$. In view of the conjugate complex property, we denote

$$
\begin{aligned}
\kappa_{1}, \kappa_{2} & =\alpha \pm i \beta=\rho e^{ \pm i \varphi}, \\
z_{1} & =1-\kappa_{1}^{2}=\left(1-\alpha^{2}+\beta^{2}\right)-i(2 \alpha \beta), \\
\bar{z}_{1} & =1-\kappa_{2}^{2}=\left(1-\alpha^{2}+\beta^{2}\right)+i(2 \alpha \beta), \\
z_{2} & =\bar{z}_{2}=2 /(1+\phi)=-2 b, \\
z_{3} & =\nu \kappa_{1} a\left(1+\kappa_{1}^{2}+2 /(1+\phi),\right. \\
\bar{z}_{3} & =\nu \kappa_{2} a\left(1+\kappa_{2}^{2}+2 /(1+\phi),\right. \\
w_{1} & =J_{1}\left(\nu \kappa_{1} a\right), \quad \bar{w}_{1}=J_{1}\left(\nu \kappa_{2} a\right), \\
w_{2} & =J_{0}\left(\nu \kappa_{1} a\right), \quad \bar{w}_{2}=J_{0}\left(\nu \kappa_{2} a\right),
\end{aligned}
$$

and

$$
A_{1}, A_{1}=B_{1} \pm i B_{2}
$$

where $\alpha, \beta, \rho$ and $\varphi$ are real variables, $B_{i}$ real constants and an overbar denotes the conjugate. Hence the characteristic equation similar to (23) reduces to

$$
\begin{gathered}
{\left[\left(z_{2} w_{1}+\overline{z_{2} w_{1}}\right)-\left(z_{3} w_{2}+\overline{z_{3} w_{2}}\right)\right] B_{1}+i\left[\left(z_{2} w_{1}-\overline{z_{2} w_{1}}\right)-\left(z_{3} w_{2}-\overline{z_{3} w_{2}}\right)\right] B_{2}=0,} \\
\left(z_{1} w_{1}+\overline{z_{1} w_{1}}\right) B_{1}+i\left(z_{1} w_{1}-\overline{z_{1} w_{1}}\right) B_{2}=0,
\end{gathered}
$$

noting that the coefficients of $B_{1}$ and $B_{2}$ are now all real, and the characteristic equation becomes

$$
\operatorname{Im}\left[\overline{z_{1} w_{1}}\left(z_{2} w_{1}-z_{3} w_{2}\right)\right]=0
$$

where Im [ ] stands for the imaginary part of [ ].

The Bessel function of a complex argument may be separated into the real and the imaginary part. We have the relations

$$
w_{k}=J_{k}\left(\nu \kappa_{1} a\right)=\operatorname{Ber}_{k}(\nu \rho a, \varphi)+i \operatorname{Bei}_{k}(\nu \rho a, \varphi) .
$$

Substituting (b) in (a), we finally obtain the condition for bifurcation:

$$
\begin{aligned}
& \frac{d_{1} \operatorname{Ber}_{1}(\nu \rho a, \varphi)+d_{2} \operatorname{Bei}_{1}(\nu \rho a, \varphi)}{d_{1} \operatorname{Bei}_{1}(\nu \rho a, \varphi)-d_{2} \operatorname{Bei}_{1}(\nu \rho a, \varphi)} \\
& \quad=\frac{d_{1}^{\prime} \operatorname{Ber}_{0}(\nu \rho a, \varphi)-d_{2}^{\prime} \operatorname{Bei}_{0}(\nu \rho a, \varphi)+2 b \operatorname{Bei}_{1}(\nu \rho a, \varphi)}{d_{1}^{\prime} \operatorname{Bei}_{0}(\nu \rho a, \varphi)-d_{2}^{\prime} \operatorname{Ber}_{0}(\nu \rho a, \varphi)+2 b \operatorname{Ber}_{1}(\nu \rho a, \varphi)}
\end{aligned}
$$


where

$$
\begin{aligned}
& d_{1}=1-\alpha^{2}+\beta^{2}, \quad d_{2}=2 \alpha \beta \\
& d_{1}^{\prime}=\nu a \alpha\left(1+\alpha^{2}-3 \beta^{2}+\frac{1}{1+\phi} \frac{2-\delta}{1+\delta}\right)
\end{aligned}
$$

and

$$
d_{2}^{\prime}=\nu a \beta\left(1+3 \alpha^{2}-\beta^{2}+\frac{1}{1+\phi} \frac{2-\delta}{1+\delta}\right)
$$

(ii) $\kappa_{1}=\kappa_{2}=\kappa$ :

(a) $\kappa$ real,

$$
\frac{\nu a\left(1-\kappa^{2}\right) J_{1}(\nu \kappa a)}{\nu a\left(1-\kappa^{2}\right) J_{0}(\nu \kappa a)-2 \kappa J_{1}(\nu \kappa a)}
$$

$$
=-\frac{2 J_{1}(\nu \kappa a)-\nu \kappa a\left[(1+\phi)\left(1+\kappa^{2}\right)+\frac{2-\delta}{1+\delta}\right] J_{0}(\nu \kappa a)}{\left[2\left(1+2 \kappa^{2}\right)(1+\phi)+\frac{2-4 \delta}{1+\delta}\right] J_{0}(\nu \kappa a)-\nu \kappa a\left[(1+\phi)\left(1+\kappa^{2}\right)+\frac{2-\delta}{1+\delta}\right]} J_{1}(\nu \kappa a) \cdot
$$

(b) $\kappa$ imaginary,

$$
\begin{aligned}
& \frac{\nu a\left(1+\chi^{2}\right) I_{1}(\nu \chi a)}{\nu a\left(1+\chi^{2}\right) I_{0}(\nu \chi a)-2 \chi I_{1}(\nu \chi a)} \\
& =-\frac{2 I_{1}(\nu \chi a)-\nu \chi a\left[(1+\phi)\left(1-\chi^{2}\right)+\frac{2-\delta}{1+\delta}\right] I_{0}(\nu \chi a)}{\left[2\left(1-2 \chi^{2}\right)(1+\phi)+\frac{2-4 \delta}{1+\delta}\right] I_{0}(\nu \chi a)-\nu \chi a\left[(1+\phi)\left(1-\chi^{2}\right)+\frac{2-\delta}{1+\delta}\right] I_{1}(\nu \chi a)} .
\end{aligned}
$$

Again (35)-(40) reduce to (24)-(27), respectively, when $h \rightarrow \infty$.

Since $h$ does not depend on the hydrostatic stress, as observed before, $p$ and $q$ appear only in the form of their difference $(p-q)$ in (35)-(40). Hence the hydrostatic pressure will have no effect on the necking or the bulging load (the load applied by the machine head) in tension or compression tests with the specimen subjected to an all-round hydrostatic pressure. This is in agreement with the conclusion reached by Hill [2], Pugh [8] and Alexander [1].

For a slender bar, $a / l \ll 1$, the critical value of $(p-q)$ is found from (35) to be

$$
(p-q)_{c} \doteq-\frac{3 \mu h}{2 \mu+h}\left(1+\frac{1}{8} \frac{n^{2} \pi^{2} a^{2}}{l^{2}}\right) .
$$

For $h \rightarrow \infty,(40)$ reduces to (29). For $\mu \rightarrow \infty$, we have

$$
(p-q)_{c} \doteq-\frac{3}{2} h\left(1+\frac{1}{8} \frac{n^{2} \pi^{2} a^{2}}{l^{2}}\right)
$$

This case is not in general identical to the case of rigid-plastic material defined by (13). With the current coordinates chosen coinciding with the principal axes of the current stress tensor, (14) does not generally approach (13) with $\mu \rightarrow \infty$ in view of the fact that in (14) when $\mu \rightarrow \infty, \epsilon_{i j} \neq 0$, for $i \neq j$. Furthermore, the inversion of (13) is nonunique (Sewell [9]). 
6. Conclusion. Axisymmetric bifurcations in an elastic-plastic cylindrical bar have been investigated as problems of existence of a nonunique solution for continuing incremental deformation from a state of finite deformation. It has been shown that necking and bulging deformations for a rigid-plastic solid are ruled out by the equations of compatibility alone, and, in all cases, bifurcation occurs only when the difference between $p$ and $q, p-q$, reaches some critical value. Hence in tension and compression tests with the specimen subjected to an all-round hydrostatic pressure, the critical load (the load applied by the machine head) is independent of the hydrostatic pressure. This is in agreement with the conclusion reached by Hill [2], Pugh [8], and Alexander [1].

Appendix. The constitutive equation for rigid-plastic material. In the constitutive equation for an isotropic rigid plastic material, it can be shown that it is immaterial whether the Jaumann derivative or the material derivative is used in the definition of the rate of stress. Taking a Cartesian coordinate system coinciding with the principal axes of the current stress tensor, we have

$$
m_{i j}\left(D \sigma_{i i} / D t\right)=m_{i j}\left(\dot{\sigma}_{i i}-\sigma_{i k} \omega_{i k}-\sigma_{i k} \omega_{i k}\right)=m_{i j} \dot{\sigma}_{i j}-\Psi
$$

where

$$
\Psi=m_{i j} \sigma_{i k} \omega_{j k}+m_{i j} \sigma_{i k} \omega_{i k}
$$

Since the principal axes of $\sigma_{i j}$ coincide with those of $m_{i j}$, both $\sigma_{i j}$ and $m_{i j}$ are diagonal tensors and so is their tensor product. Besides, since $\omega_{i}$ is skew symmetric, the term $m_{i j} \sigma_{i k} \omega_{j k}$ in (A2) vanishes, and by the same reasoning, $m_{i j} \sigma_{i k} \omega_{i k}$ also vanishes. Therefore we have $\Psi=0$ and consequently $m_{i j}\left(D \sigma_{i j} / D t\right)=m_{i j} \dot{\sigma}_{i i}$. Certainly this is also true for any general coordinate system, so that

$$
m_{i i}\left(D \sigma^{i j} / D t\right)=m_{i}, \dot{\sigma}^{i i}
$$

remembering that $m_{i}$ are now the components referred to the general coordinate frame.

\section{REFERENCES}

[1] J. M. Alexander, The effect on tensile plastic instability-a fallacious argument, J. Inst. Metals 93, 366-367 (1965)

[2] R. Hill, On the problem of uniqueness in the theory of a rigid-plastic solid. III, J. Mech. Phys. Solids 5, 153-161 (1957)

[3] R. Hill, $A$ general theory of uniqueness and stability in elastic-plastic solid, J. Mech. Phys. Solids 6, 236-249 (1958)

[4] R. Hill, Uniqueness crileria and extremum principles in self-adjoint problems of continuum mechanics, J. Mech. Phys. Solids 10, 185-249 (1962)

[5] A. D. Kerr and S. Tang, The effect of lateral hydrostatic pressure on the instability of elastic solids, particularly beams and plates, J. Appl. Mech. 33, 617-622 (1966)

[6] A. D. Kerr and S. Tang, The instability of a rectangular elastic solid, Acta Mech. 4, 43-63 (1967)

[7] W. Prager, Three-dimensional plastic flow under uniform stress, Rev. Sci. Univ. Istanbul (A) 19, 23-27 (1954)

[8] H. L. Pugh, The mechanical properties and deformation characteritics of metals and alloys under pressure, First Conference on Materials, A. S. T. M., 1964

[9] M. J. Sewell, Inverse rigid/plastic constitutive equations, Internat. J. Engrg. Sci. 2, 317-325 (1964)

[10] Z. Wesolowski, The axially symmetric problem of stability loss of an elastic bar subject to tension, Arch. Mech. Stos. 15, 383-395 (1963)

[11] C. H. Wu and O. E. Widera, Stability of a thick mubber solid subjected to pressure loads, Int. J. Solids Structures 5, 1107-1117 (1969) 